\title{
Les groupes Facebook comme dispositif de médiation patrimoniale du music-hall à Barcelone
}

Facebook groups as dispositives of mediation of music hall heritage in Barcelona

Irene Gallego

\section{CpenEdition}

Journals

Édition électronique

URL : http://journals.openedition.org/edc/6461

DOI : $10.4000 /$ edc.6461

ISSN : 2101-0366

Éditeur

Université Lille-3

Édition imprimée

Date de publication : 1 décembre 2015

Pagination : $35-52$

ISBN : 978-2-917562-14-7

ISSN : $1270-6841$

Référence électronique

Irene Gallego, «Les groupes Facebook comme dispositif de médiation patrimoniale du music-hall à Barcelone ", Études de communication [En ligne], 45 | 2015, mis en ligne le 01 décembre 2017, consulté le 19 avril 2019. URL : http://journals.openedition.org/edc/6461 ; DOI : 10.4000/edc.6461

(c) Tous droits réservés 


\section{Les groupes Facebook} comme dispositif de médiation patrimoniale du music-hall à Barcelone

Facebook groups as dispositives of mediation of music hall heritage in Barcelona 
Résumé / Abstract

Cet article cherche à montrer comment Facebook est approprié et utilisé comme dispositif de médiation patrimoniale par un ensemble de passionnés du music-hall à Barcelone, dans la mesure où les modes d'usages répondent à leurs désirs mémoriaux et à leur quête de reconnaissance. Dans les appropriations et les usages de cette plate-forme numérique, on retrouve des représentations et des formes de sociabilité caractéristique du monde du music-hall à Barcelone. Pourtant, ses nouvelles manifestions sont toujours façonnées par les contraintes techniques et les usages conventionnels du dispositif de médiation.

Mots-clés : Reconnaissance, valorisation, patrimoine, groupes Facebook, music-hall, Barcelone.
The purpose of this article is to show how Facebook is appropriated and used as a dispositive for heritage mediation by a group of music hall fans in Barcelona, and especially how modes of use correspond to fans' desire for memorialization and their quest for recognition. Among appropriations and uses of this social networking site we find representations and forms of sociability that are characteristic of the world of music halls in Barcelona. However, these new manifestations are always conditioned by technical constraints and by conventional uses of the communication dispositive.

Keywords: Recognition, valorization, heritage, Facebook groups, music hall, Barcelona. 
$\times \quad J$ 'aimais bien El Molino classique et comme j'ai grandi dans la rue Rosal, que grâce à ma mère, j'ai connu beaucoup d'artistes, que j'ai eu trois copines danseuses, et que ma cousine était aussi du corps de danse, j'ai donc décidé de créer le groupe (Paco, conversation en ligne, 19-05-2014).

Établissement à capitaux privés, mais vécu comme un bien commun, El Molino était une institution barcelonaise dédiée aux variétés, longtemps considérée comme l'emblème d'El Paralelo, cet univers de music-hall fréquemment comparé avec Montmartre ou Broadway'. Paco, internaute assidu et membre de Facebook depuis ses débuts, ouvre en novembre 2009 le groupe «El Molino de Barcelona. Official club de fans ". II met comme photo de profil ${ }^{2}$ une image d'une célèbre aquarelle réalisée en 1987 par un peintre local, représentant la façade du café-concert. En dessous, Paco poste un bref historique sur ce café-concert et sur El Paralelo, qu'il a bricolé à l'aide de livres sur celui-ci, de la mémoire collective et de ses propres souvenirs. Cette initiative, comme d'autres groupes qui se sont créés entre 2009 et 2013, rend hommage à ceux qui dans le passé l'ont fait exister et se veut comme un espace où les passionnés d'El Paralelo peuvent partager des photographies, des souvenirs, etc.

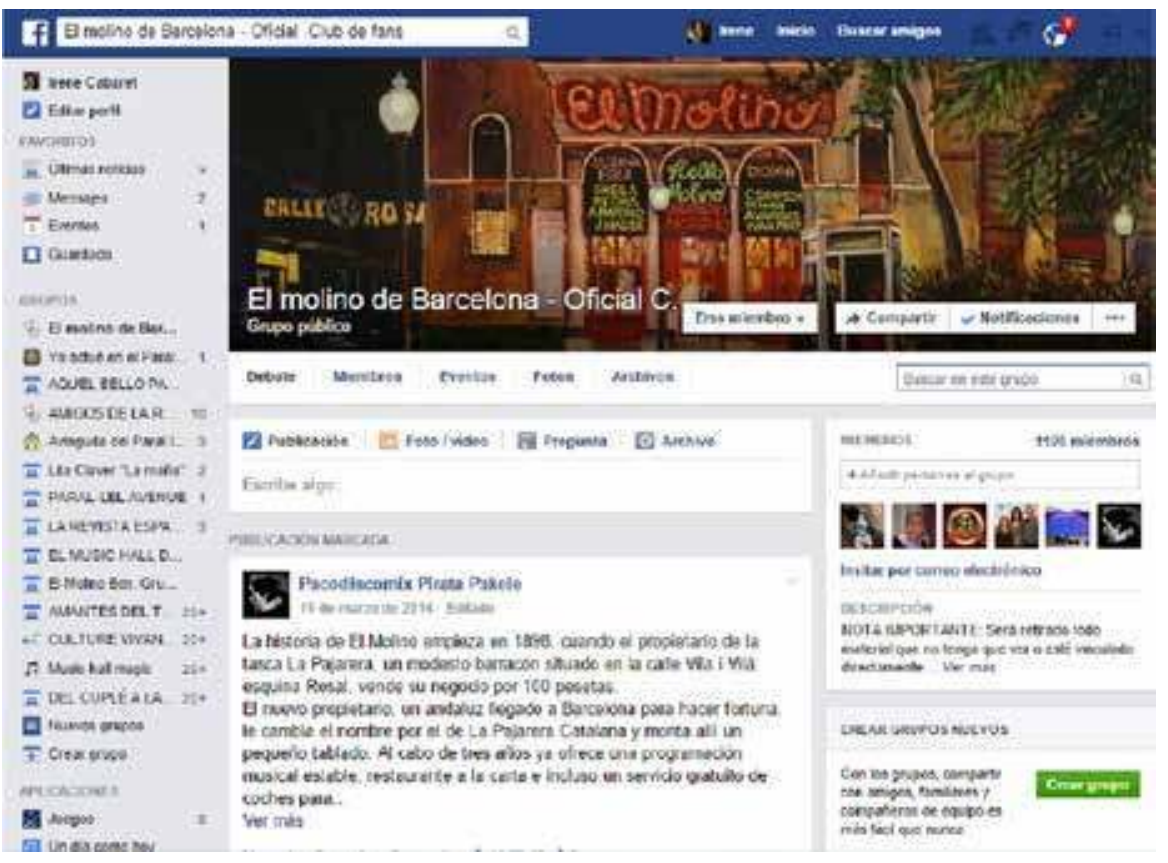

Figure 1: El Molino de Barcelona-Oficial Club de fans

1 Ce monde s'est développé autour de l'Avenida del Paralelo, inaugurée en 1894 dans un quartier de Barcelone.

2 La photo de profil est celle qui apparaît en permanence et en tête de la page du groupe Facebook. Elle sert à caractériser le contenu de la page et à lui donner une illustration. 
Engagée depuis 2013 dans une enquête ethnographique sur les transformations pratiques et symboliques du monde du music-hall à Barcelone depuis 1980, je me suis confrontée à des usages intenses sur les plates-formes participatives (blogs, Facebook, YouTube) où des internautes font ressortir du passé ce monde révolu tout en le valorisant. Plus particulièrement, la prégnance de Facebook au quotidien et les conversations de mes informateurs m'ont amenée à m'y intéresser de plus près. "Tout le matériel d'El Molino est bienvenu et considéré ici comme des joyaux». Ce créateur d'un groupe résume bien ici l'esprit des groupes, ainsi que les enjeux inhérents à leurs dynamiques, me permettant de fonder mon hypothèse de travail.

Si El Paralelo a été longtemps déclassé et négligé par les institutions culturelles légitimes, sur les groupes Facebook ses traces sont plutôt perçues comme des objets de valeur, ou comme l'affirme le créateur ci-dessus, des joyaux. Ce changement de valeur opéré sur les plates-formes virtuelles permet d'envisager les groupes Facebook comme un dispositif de médiation patrimoniale. On peut ainsi se demander comment procèdent ces usagers afin de mettre en valeur El Paralelo? Quels sont les objets partagés et les critères qui président aux choix de partage?

Ce phénomène nous place au cœur de ce que Daniel Fabre appelle «l'âge du patrimoine». Selon lui, ce qui différencie «le moment du patrimoine des modes d'institution de la culture qui l'ont précédé tient à la présence nécessaire, à la forme renouvelée, à l'impact et, surtout, à l'extension très amplifiée des émotions qui le caractérisent » (Fabre, 2013, 22). En fait, ce lien émotionnel qui relie les usagers à El Paralelo est à l'origine de leur mobilisation. Leurs pratiques de médiation seront analysées à partir d'une approche pragmatique qui spécifie "les ressources des personnes et les compétences à parler et agir dans l'espace public qu'elles requièrent » (Tornatore, 2006, 516). Les nouveaux agencements dont fait objet El Paralelo doivent aussi être analysés à partir de «la dimension d'un faire croire qui lui est pourtant consubstantiel et qui est la condition de son efficacité symbolique» (Fabiani, 2007, 12). Afin de comprendre dans toute leur complexité les médiations nous envisagerons ainsi ce que les documents publiés font chez les membres, quels sont les émotions et les souvenirs suscités, aussi bien que ce que le dispositif leur fait faire, les contraintes techniques et morales. L'approche pragmatique «redonne leur place aux objets comme aux sujets en tant que les uns et les autres agissent, au lieu de les considérer comme des supports passifs de projection» (Heinich, 2009, 36).

Pour récolter mes données de terrain, j'ai procédé par l'observation en situation. J'ai analysé les situations d'échange entre membres de plusieurs groupes Facebook ${ }^{3}$ sur trois années. L'observation était participative dans la 
mesure où j'en étais aussi membre et que je publiais également des contenus et exprimais des impressions. Parfois, les échanges avec les membres ont été réalisés en privé via le chat. Cet outil a été précieux pour établir un rapport de proximité avec quelques personnes. Afin de mieux appréhender les procédés des acteurs, les principes et motivations de leurs pratiques, j'ai par ailleurs réalisé plusieurs entretiens thématiques (20h environ), in situ ou par téléphone, avec les membres les plus actifs. Enfin, la participation à plusieurs événements relayés par les groupes a été une autre source importante d'observation.

1

\section{L'émergence des groupes Facebook dans un contexte de "revitalisation »}

Entre la fin de la décennie 1990 et le début des années 2000 à Barcelone, El Paralelo a connu de profondes transformations. La fermeture des derniers établissements de variétés tels qu'El Barcelone de Noche, El Molino ou El Teatre Arnau, ainsi qu'un changement dans la programmation des théâtres encore actifs - orientés désormais vers la comédie et la comédie musicaleinstaurent une forme de rupture dans la conception traditionnelle qui reliait $E I$ Paralelo avec le monde du music-hall. Depuis 2010, une série d'événements a fait ressortir du passé le music-hall ou l'ancien El Paralelo. La réouverture d'El Molino en 2010, avec une programmation mélangeant les variétés d'autrefois et le new-burlesque, semble être le catalyseur d'un phénomène qu'on qualifie de «revitalisation».

Après une décennie de portes closes et de dégradation continue, El Molino est racheté en 2007 par une entreprise privée nommée Ocio Puro. La fin d'El Paralelo ayant été marquée par une série de faits vécus comme humiliants ${ }^{4}$, la nouvelle de sa réouverture et d'autres initiatives de redynamisation suscitent l'émotion et semblent vécues comme le dernier avatar de cette série. La participation des anciens artistes s'avère toutefois très faible. À la suite de leurs réactions, on peut dire que la «revitalisation» d'El Paralelo instaure une rupture avec leur tradition professionnelle tout en donnant lieu à un sentiment de dépossession et d'invisibilité. Ce qui est vécu par eux comme du mépris social devant être réparé. En outre, la réhabilitation d'El Molino ne déçoit pas seulement les anciens professionnels, mais une grande partie des Barcelonais. Aux critiques envers sa gestion économique et artistique- il ne plaît ni aux anciens passionnés ni aux nouveaux publics-, s'ajoute une autre critique

4 Comme par exemple le fait de comparer El Molino à un stade de football, l'évacuation forcée de ses travailleurs du site et la découverte de ses archives et matériaux scéniques jetés dans les containers publics de poubelles ou I'incendie du Teatro Talia. Ces quelques épisodes scandent la fin d'El Molino et constituent des points d'articulation des récits autour de celui-ci. 
plus répandue qui dénonce le fait d'avoir détruit un bâtiment à tous les égards emblématique. Le démantèlement d'El Molino ne renvoie pas seulement à sa dimension symbolique, ni architectural, mais aussi à une sorte d'arrachement de la capacité du lieu à incarner le passé et à faire se souvenir. Son remplacement par un bâtiment perçu comme un «non-lieu» (Augé, 1992) génère un certain malaise parmi les habitants, qui impulse dès lors la dynamique des groupes Facebook, ainsi que d'autres mobilisations pour la valorisation d'El Paralelo.

Dans le sillage de ces événements marquants, les usages que les membres font des plate-formes peuvent être interprétés à la suite de Ricœur comme un travail de justice mémorielle, où «c'est la justice qui, extrayant des souvenirs traumatisants leur valeur exemplaire, retourne la mémoire en projet; et c'est ce même projet de justice qui donne au devoir de mémoire la forme de futur et de l'impératif » $(2000,105)$.

\section{2 \\ De la culture virtuelle vers la médiation patrimoniale}

Les plates-formes participatives web 2.0, même si elles sont des initiatives individuelles, n'existent que grâce à une collectivité d'usagers articulée par des «liens faibles» (Granovetter, 1973) qui y participe et leur donne un certain dynamisme. Au sein de celles-ci, l'interchangeabilité entre les positions d'éditeur et de lecteur ouvre la possibilité à un projet collaboratif et collectif. Ainsi n'importe quel membre peut à la fois observer et réaliser une publication. Les différents groupes étudiés ont été créés par d'anciens fans ou artistes qui ont pris le rôle d'administrateur ${ }^{5}$. Cependant ces groupes sont conçus comme des projets ouverts à tous, indépendamment du statut professionnel ${ }^{6}$. Le nom et la photographie du groupe, ainsi que certaines informations consignées sur I'interface sont des mises à distance qui permettent de souligner la dimension participative du projet («club», "groupe»), l'égalité entre les membres et le lien émotionnel («amis», "fans»). Les membres participent en général à plusieurs groupes ce qui donne lieu par ailleurs à une circulation horizontale des contenus et participe d'une identité commune.

5 Membre censé gérer et contrôler les publications; il a entre autre la possibilité d'effacer des contenus et d'éliminer des membres ayant des comportements irrespectueux. Les membres les plus actifs peuvent aussi devenir administrateur, un poste réputé qui se mérite et donne une certaine autorité.

6 Il y a en effet d'autres groupes autour d'El Paralelo qui sont conçus plus spécifiquement pour des anciens travailleurs, caractéristique qui est mise en avant dans le nom «El Molino Bcn. Grupo de ex-Molineras», "Yo actué en el Paralelo barcelonés». 
Actuellement, chacun des groupes compte au moins 500 membres $^{7}$, dont l'âge moyen dépasse souvent les 50 ans. À cet égard, les retours sur publication semblent assez faibles: le nombre de «Like» ne surpasse pas la vingtaine et les échanges textuels n'engagent rarement plus de dix personnes. Ces actions, ainsi que les publications, sont en fait réalisées par une petite minorité très active (Cardon, 2008) ${ }^{8}$, les "vrais " passionnés, qui agit comme médiatrice entre le passé et le présent, entre la collectivité et le groupe (Bensa, 2001). Son investissement en temps est très significatif. Outre le temps propre au visionnage de documents et aux interactions avec les membres, cette minorité consacre un nombre considérable d'heures à numériser leurs collections, à s'informer et à chercher des documents susceptibles d'être partagés avec le groupe. Ces actions impliquent une certaine maîtrise des nouvelles technologies, aussi bien qu'une familiarisation avec le groupe. Bien que Facebook ait permis l'actualisation d'anciennes connaissances - et dans quelques cas il s'agit d'amitiés préalables - le groupe confronte chaque usager à une collectivité large d'inconnus ce qui donne aux échanges une dimension publique. Les groupes instaurent une situation de communication et un régime singulier de visibilité qui peut donner lieu autant à des formes de reconnaissance que de vulnérabilité. On entend par reconnaissance l'expression publique, par des actes et des gestes appropriés à la situation et au rapport social, du fait qu'une personne est censée posséder une «valeur» sociale (Honneth, 2005).

L'engagement de cette minorité ressemble à l'acquittement d'une dette envers autrui (Ricœur, 2000, 108), de fils envers ses parents, de spectateurs envers leurs idoles ou d'élèves envers leurs maîtres. Ce qui peut être vu, à la suite de Honneth, comme une forme de reconnaissance propre à la sphère de l'amour ou de la socialité primaire (Caillé, 2007, 10). Or, dans la mesure où la plate-forme vise la valorisation d'El Paralelo comme bien commun, ce qui est en jeu c'est «l'estime au prorata de [leur] contribution productive» (Ibid.) au monde du music-hall et à son histoire. Le déplacement de la reconnaissance de la sphère de la sociabilité primaire vers celle de la division du travail, implique d'abord une mise à distance des membres "endettés", permettant aussi de transformer leurs engagements personnels en projet collectif. D'un autre côté, dans la mesure où le groupe se développe, celui-ci devient aussi un bien commun à entretenir. Les publications et les échanges ne sont pas seulement une forme de justice envers le passé, ils répondent aussi à un engagement

7 «El Molino de Barcelona » et "Lita Claver "La Maña"» comptent par exemple plus de mille membres.

8 Bien que les membres ne mobilisent pas une terminologie particulière pour parler de cette minorité aux contours flous, il existe toutefois des formes de reconnaissance grâce au rapport réflexif que les usagers entretiennent avec le groupe. 
avec le groupe ${ }^{9}$, ce qui implique d'ajuster les intérêts individuels aux intérêts collectifs ${ }^{10}$.

Si le groupe Facebook se voit comme un moyen effectif pour favoriser la diffusion et la valorisation d'El Paralelo, il génère toutefois une série de tensions qui peuvent mettre en péril les formes de reconnaissance inhérentes à ce projet. Certaines tensions sont propres à la culture du numérique et aux dispositifs web 2.0 comme les pratiques de braconnage ${ }^{11}$ par exemple, alors que d'autres relèvent de la spécificité de ce monde du spectacle et de la restitution de ses traces. La nudité des artistes et le respect de leur intimité apparaît par exemple comme un des enjeux majeurs. Ces différentes tensions semblent imbriquées et sont négociées en situation par les usagers de Facebook. L'interconnaissance préalable ou établie grâce au groupe, aussi bien que l'expérience sur le site permettent aux membres d'avoir des références sur la personnalité des autres membres et leur rapport aux objets-images, ainsi qu'une idée sur la façon dont les artistes vivent leur passé et rendent leur vie publique. Plus que des normes explicites, une forme de morale diffuse et de «vigilance participative» (Cardon, Levrel, 2009) gère les groupes. Cette morale résulte de négociations constantes entre les attentes collectives (l'ethos du groupe) et les éthiques individuelles. La réprobation ou la négociation envers des attitudes et des actions considérées comme déplacées peuvent se déployer publiquement sur le site ou à travers des échanges privés. S'il n'y a pas de consensus les administrateurs sont alors sollicités.

La réparation d'une mémoire empêchée et le travail de justice prend donc sur Facebook la forme d'une intense dynamique de partage autour de la trace et de la mémoire. Cette dynamique se développe avec sa propre temporalitéqui est celle de ses publications - sans se soucier d'une restitution critique ou chronologique des documents. Au fil du temps les groupes se sont stabilisés en désignant les contours de ce qui est susceptible d'être partagé, c'est-à-dire ce qui doit être montré et ce qui est de l'intérêt du groupe.

\section{3}

\section{La mise en valeur du monde du music-hall}

Selon Fabre, la mise en valeur consiste à «mettre entre guillemets un certain nombre de choses, matérielles et immatérielles, qui sont donc, par ce

9 Les usagers utilisent souvent cette expression: "lo prometido es deuda», "la promesse est une dette».

10 Ce point de discussion autour des enjeux inhérents aux différentes formes de reconnaissances imbriquées dans les dynamiques de Facebook mériterait une étude approfondie qui n'aurait pas lieu d'être ici.

11 Appropriation de documents mettant en exergue le contrôle sur la propriété légale ou symbolique. 
fait même, transférées de leur régime d'existence antérieur à un régime autre, à vrai dire radicalement nouveau » $(2013,22)$. Cela n'implique pas forcément un changement du sens que possèdent ces objets (Ibid.). Concernant les pratiques des usages Facebook à propos d'El Paralelo, on peut se demander quelles sont les «choses" valorisées et sur la base de quels choix celles-ci sont partagées.

Pour qu'une plate-forme participative existe dans la longue durée, une dynamique constante d'édition de contenus semble nécessaire, ce qui peut brouiller parfois les limites entre ce qui est susceptible d'être partagé ou non. Cette tension correspond à l'alternative entre conserver ou écarter, transversale à toute entreprise patrimoniale (Heinich, 2009, 233) et semble mettre en exergue l'acte même de valorisation. Au-delà des contraintes morales des groupes, qui donnent parfois lieu à des actes de censure, l'analyse montre que, loin de tout publier, une série de principes et de critères guident le choix des usagers. Leurs critères renvoient à des "prises", ce qu'on entend à la suite de Heinich comme «le point de rencontre entre les propriétés objectales des éléments soumis au jugement et les ressources des acteurs amenés à juger [...]. Pour s'emparer de ces prises et en faire usage, les acteurs disposent des ressources préalablement constituées et relativement partagées, qui prennent la forme d'un nombre fini de 'critères'» $(2009,234)$. Les ressources étant ici la maîtrise des technologies numériques et la bonne connaissance d'El Paralelo, dont le partage fonde l'ethos de groupe.

Les critères tels que la qualité graphique ou audiovisuelle, la valeur documentaire ou la nature de document (artistique, commerciale, journalistique) semblent peu importer. Les choix se font plutôt en fonction des choses représentées et du rapport personnel à cette chose. Les pratiques de recherche sur Internet et la mise en numérique des collections privées répondent en effet aux liens affectifs anciens avec El Paralelo et s'inscrivent dans l'actualité locale. Par ailleurs, l'engagement avec le groupe ou plus particulièrement avec certains membres influe également sur le degré d'investissement de certains. En fait, ceux-ci mobilisent une vision d'ensemble et un suivi de ce que les autres usagers font. Ainsi chaque nouvelle publication prend en compte le travail déjà accompli (par soi-même et par les autres) ainsi que les informations fournies par les commentaires et des sollicitations telles que celle-ci: «Imanol Alvarez [membre], comme tu cherches des couvertures de revues, je t'offre ces deux là... Salut» (membre-ancien artiste, commentaire FB, 06-12-2014).

La cohérence thématique - faire partie d'El Paralelo- semble être le critère le plus important. Les membres mobilisent ce critère de façon très inclusive sans trop se soucier des hiérarchies qui existaient au sein de ce monde. Aux côtés des grandes vedettes et des hauts lieux, d'autres salles, artistes et travailleurs plus modestes trouvent leur place au sein de ces groupes. Chacun y est envisagé dans sa propre singularité. Les membres publient aussi parfois des photographies d'établissement dont leur inscription remet en question le périmètre d'El Paralelo. À l'instar de certaines photographies de vedettes 
ayant travaillé dans ces établissements. Le critère thématique et son caractère flexible déplace la problématique de la dimension axiologique vers sa dimension ontologique : qu'est ce qu'El Paralelo? Quel est son contour et sa définition?

Après une publication à propos d'un livre sur l'industrie de la mode en Catalogne (17-04-2015), l'administrateur du groupe «Aquel bello Paral-lel» écrit : "Ce site est seulement pour des choses et des événements en lien avec El Paralelo. Merci ». L'auteur de la publication répond «à El Paralelo il y avait aussi des usines». Cet échange met en relief qu'un "objet culturel peut être l'objet de prises multiples, et donc de compréhensions diverses au sein d'un même espace de signification" (Fabiani, 2007, 10). En fait El Paralelo, en tant que monde du music-hall, est une construction; il n'existe pas au-delà du geste qui le prend pour objet et le fait advenir. Les membres envisagent ce monde à partir de leurs expériences passées et l'objectivent à l'aide d'un ensemble d'images, de vidéos, de chansons et d'écritures. L'ancrage dans l'expérience privilégie des contenus qui renvoient au temps du vécu, c'est-à-dire à la période comprise entre 1970 et aujourd'hui.

\subsection{La topique d'El Paralelo}

Malgré leur diversité, les contenus ne renvoient qu'à trois caractéristiques principales: les «lieux», les «spectacles» et les "artistes». Même si elles sont toutefois fortement imbriquées, elles illustrent différentes manières de se relier à El Paralelo et de fonder sa valeur.

Ainsi, L'Avenida del Paralelo et différents établissements de spectacle (théâtres, café-concert et cabarets) ou de sociabilité (bars et restaurants) constituent un premier lieu de discours. Ces lieux vécus comme des "propres» constituent des cadres de vie et de travail. Cependant ce sont leurs aspects architecturaux et historiques qui permettent de fonder la valeur patrimoniale d'El Paralelo. «En 1910, la construction originelle [du Molino] faite en bois a été démolie donnant lieu à une réhabilitation par l'architecte moderniste barcelonais Manuel Joaquim Raspall (disciple de Domènech i Montaner)». 


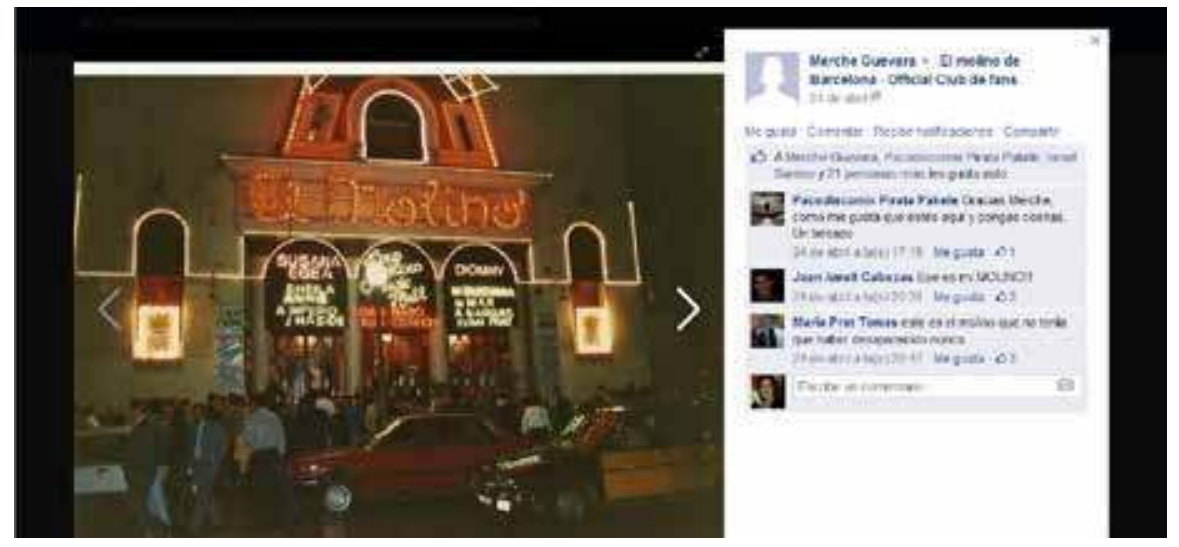

Figure 2: L'ancien Molino

Un deuxième lieu de discours correspond aux «spectacles». II fait référence à l'ensemble des événements lyrico-scèniques s'étant déroulé dans les lieux. Ces occurrences performatives procurent des émotions, du plaisir esthétique, qui participent à la consolidation d'un goût pour le music-hall, d'un attachement particulier. Les spectacles introduisent une autre façon de caractériser El Paralelo, à travers sa dimension immatérielle, même si c'est le recours aux valeurs du monde du théâtre- le genre scénique comme catégorie générique - qui le fonde comme bien commun: "Le Music Hall... la revue... le cabaret... un genre fantastique et libre, un monde de gaieté et de couleur, un état d'esprit qui frôle la folie et la bonne humeur » (membre, commentaire FB, 30-06-2009).
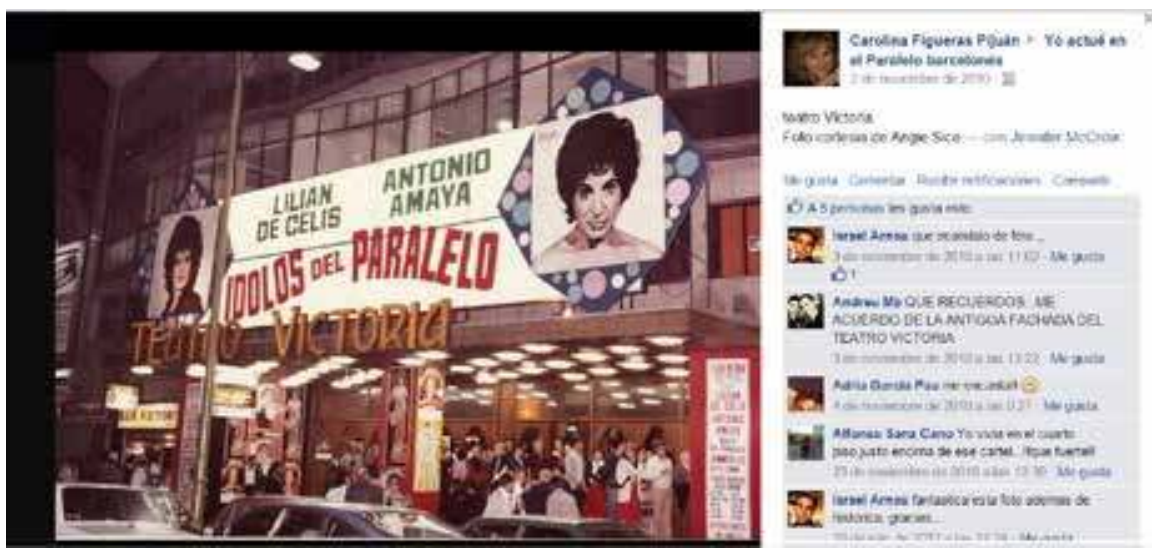

Figure 3: Spectacle «Idolos del Paralelo» au Teatro Victoria

Le troisième lieu de discours correspond à l'ensemble des vedettes, comédiens, danseuses et danseurs qui ont mis en scène ces spectacles dans ces lieux spectaculaires, et dans une moindre mesure, à d'autres travailleurs 
(entrepreneurs, serveurs, couturiers, compositeurs, etc.). Ce sont des "artistes» avec lesquels les membres ont établi des liens notamment d'admiration, mais aussi des rapports professionnels et d'amitié. Leur savoir-faire témoigne de leur excellence ou de leur singularité et ces artistes sont investis en ligne d'une valeur de célébrité.

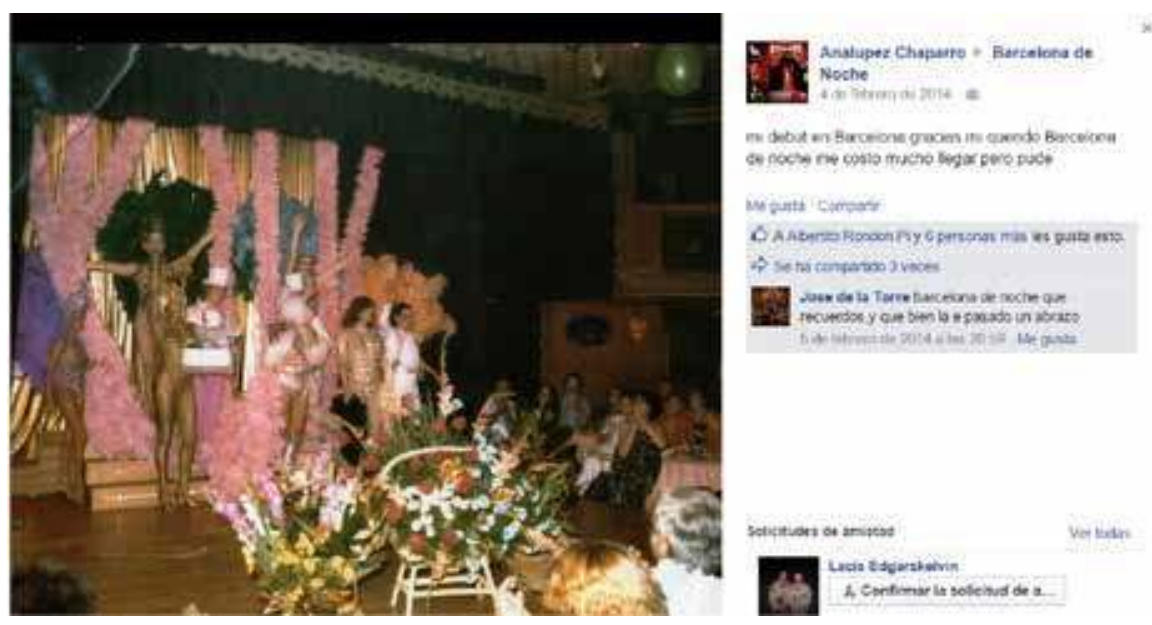

Figure 4: Artistes du cabaret Barcelona de Noche

Ainsi, la mise en valeur d'El Paralelo se fait au travers de manipulations (techniques et discursives) d'objets et de documents visant ces trois lieux de discours. Les publications, ainsi que les jeux interprétatifs et mémoriels qu'elles déclenchent donnent lieu à ce qu'on peut appeler la topique d'El Paralelo, entendant par là «ces 'ontologies' ordinaires, en généralité et communes - mais qui n'excluent pas un principe de variabilité- qui se confectionnent dans la déclinaison commune de lieux thématiques obligés " (Cheyronnaud, 2006, 44). À l'égard de ces trois aspects, les attachements des membres répondent à la fois à des liens affectifs envers les lieux, à des liens émotionnels envers les personnes et à des liens esthétiques envers le music-hall. Dans la mesure où le groupe envisage El Paralelo comme un bien commun, les pratiques autour de la plate-forme se développent comme un jeu constant de mise à distance et d'appropriations.

\subsection{Les pratiques de documentation}

Les documents partagés au sein des groupes proviennent en partie de collections privées ${ }^{12}$. Au-delà de ces collections, Internet constitue l'autre grande

12 Parmi les documents, on trouve entre autres, programmes de salle, affiches, disques, livres, revues, coupures de presse, boîtes d'allumettes, verres des établissements, photographies dédicacées, enregistrements vidéo, partitions, livrets, perruques, décors, habits, etc. 
ressource documentaire. La recherche sur la toile permet aux usagers d'élargir leurs collections ${ }^{13}$ et leurs connaissances autour d'un monde connu et vécu, mais aussi de se plonger rétrospectivement vers les époques précédant les générations des membres les plus âgés. Ils s'approprient ainsi des documents venant de sites très variés, qui parfois n'ont pas de lien direct avec le monde du spectacle ou avec El Paralelo-par exemple des sites des brocantes ou des sites consacrés à Barcelone-, ou alors ils publient des hyperliens de sites web tels que des journaux, des blogs ou des vidéos YouTube qui hébergent des publications sur le sujet. La recherche sur Internet entraîne ainsi une plus grande hétérogénéité dans la nature et le contenu des documents partagés.

Les contenus choisis sont mis en forme. II s'agit d'une opération de médiation qui entraîne une série de choix et de procédés, de jeu d'échelles et de dimensions. Ces manipulations entraînent des transformations significatives sur la matérialité de l'objet, aussi bien que sur sa nature ontologique et sur son statut. Afin de produire une image, les membres procèdent à différentes prises de vue ${ }^{14}$, sélectionnent des parties et combinent différentes images entre ellessuivant normalement une logique thématique tel qu'un même artiste, spectacle ou lieu. Ces images ainsi produites apparaissent comme des indices d'un ensemble d'objets et de documents, mais certains procédés rendent toutefois difficile leur identification. Par exemple, dans le cas des programmes de salle ou des revues érotiques, la mise en image donne lieu à une forte transformation de la nature de l'objet. L'encadrement de l'image de l'artiste et l'élimination des aspects "secondaires» (publicité, texte) n'implique pas seulement une hiérarchie entre les composantes du document, mais aussi une préférence pour certains objets: ici le portrait plutôt que la revue ou le programme. En ce qui concerne les spectacles enregistrés ou les films musicaux, certains les fractionnent en séquences ou en numéros et réalisent des assemblages qui les recomposent. Avec ce type de procédé, la partie est mise en avant face à la totalité. L'écoute et le visionnage sur Facebook ou sur YouTube se base sur des unités minimales de sens, de mémoire et de durée. On n'est plus face à un spectacle, ni face à un film, mais plutôt face à des situations performatives significatives. Ces unités sont façonnées par les usages conventionnels de ces plate-formes et par leurs contraintes techniques, même si celles-ci reposent sur une structure préalable qui renvoie à celle du spectacle ou du film musical ${ }^{15}$.

13 Ils stockent des contenus sur leurs ordinateurs ou sur leurs comptes personnels Facebook.

14 Dans le cas des objets, ils suivent, si on peut dire, le canon muséal (prise de vue frontal et fond décontextualisé) ou alors ils réalisent une copie numérique, dans le cas de documents.

15 Le spectacle de «revue» est composé d'une suite de numéros divers (ouverture, numéro de danse, cirque, chant, sketchs, bouquet final) développés chacun autour d'une chanson. Ces numéros ont été repris et recombinés au fil du temps ce qui a facilité leur mémorisation plutôt que celle du spectacle en entier. 
Les documents ainsi produits présentent un caractère impersonnel, puisque le lien qui les relie avec son médiateur, aussi bien que la présence de celui-ci, sont absents. Or, ces documents, loin d'être des traces objectives d'un monde révolu, relèvent d'un regard particulier sur El Paralelo, à la fois de celui qui les a créés et de ceux qui les manipulent ensuite. Les documents ainsi retravaillés privilégient la perspective du spectateur ordinaire. Sur le plan spatial, elle se déploie de l'extérieur vers l'intérieur, du parterre vers la scène et de la rue vers le théâtre. Cette perspective qui présuppose un admirateur regardant un artiste, un spectateur prêt à la joie d'un spectacle, se déploie, au sens symbolique, de l'ordinaire vers l'extraordinaire ${ }^{16}$.

La publication de documents s'accompagne de notes d'ampleur variable, allant de la simple légende- nature de l'objet, nom de l'artiste, lieu, date, titre du spectacle- jusqu'à des descriptions et des mises en contexte beaucoup plus élaborées telles que des notices biographiques et historiques. Toutefois, les notes au-dessus d'une certaine taille doivent être déroulées par le lecteur pour être lues. En ce sens la plate-forme tend plutôt à privilégier la visibilité des documents iconographiques. Dans cet exercice d'écriture les membres masquent leur présence en tant qu'énonciateur et intègrent des formes de généralité telles que l'histoire et l'imaginaire partagés d'El Paralelo ou des passages de la critique théâtrale. La présentation «démodalisée» du document (Cardon et al., 1995, 13) sert à la valorisation d'El Paralelo, même si cependant, on constate parfois des implications à la première personne qui atténuent l'excès de distanciation critique et correspond mieux aux attentes d'un groupe de fans.

Quand les contenus peuvent être ambivalents, comme dans le cas de photographies de nu ou les revues érotiques, les précisions et les qualificatifs employés permettent de rendre plus explicite l'intention de l'auteur - rendre hommage - tout en favorisant de nouvelles interprétations. L'écriture sert également à mettre en situation la trace tout en reliant le passé et le présent d'El Paralelo: «En regardant les milliers de CD que j'ai, regardez ce qu'il est apparu!»; «Photographie donnée par la gentillesse de sa petite-fille», etc.

Les membres mettent à l'épreuve les contenus partagés afin de confirmer qu'ils répondent aux attentes du groupe. Ainsi, la réception donne lieu à des jeux d'interprétation et de mémoire qui prennent la forme d'échanges textuels $^{17}$. Si les choix qui précèdent la publication semblent être peu restrictifs, la réception montre que toutes les publications n'ont pas la même agency,

16 Il existe cependant une petite quantité de documents qui reproduisent des photographies artistiques et journalistiques, ou même de tableaux ou des documentaires, et qui introduisent un autre regard sur ce monde, qui cherchent à montrer l'envers du music-hall, ses aspects professionnels, sociaux et leurs rouages.

17 De même que pour les notes, les commentaires et les échanges sont masqués sur la plate-forme au delà d'une certaine taille et doivent être déroulés par le lecteur intéressé. 
c'est-à-dire la même capacité à mobiliser les usagers. Les photographies des artistes les plus célèbres suscitent plus d'intérêt, par exemple. Le retour sur publication - cliquer sur "j'aime» ou laisser un commentaire- est une manière d'entretenir au présent un lien personnel entre soi, l'objet et le monde du music-hall. Ce lien peut aussi s'inscrire dans le temps par le récit d'expériences passées. La manifestation des émotions, des opinions ou l'écriture de souvenirs et d'anecdotes apparaissent comme des formes d'affirmation de soi qui permettent en même temps d'être reconnu comme participant de ce monde. Si les échanges textuels permettent l'expression d'autres voix que celles des artistes (de fans, de travailleurs et de leurs fils), cela n'implique pas pour autant d'autres perspectives dans la mesure où, généralement, ils reconduisent la doxa d'El Paralelo, «au sens de discours partagé qui a trait à la considération d'une chose, d'un lieu, etc. et dont l'autorité tiendrait d'abord dans l'insistance entre générations, à rapporter ou à reproduire» (Cheyronnaud, 2011, 5).

\section{4}

\section{Quelles médiations pour quel patrimoine?}

Les médiations rendues possibles par ces groupes Facebook sont le résultat d'une négociation entre le passé, sous la forme des traces et de dispositions (émotionnelles, esthétiques, mémorielles), et le présent qui les actualise, entre des possibilités et des contraintes. Les groupes semblent s'être stabilisés au fil du temps tout en proposant un regard particulier sur El Paralelo.

Les actions des membres s'articulent autour de trois lieux de discours imbriqués, où ils donnent à voir une grande diversité de documents, mais avec des éléments récurrents. Ce principe est sans doute le leitmotiv qui fonde l'ethos d'El Paralelo et que beaucoup de journalistes ont pointé. Ainsi, la formule «que tout varie pour que rien ne change » qui régissait l'organisation des spectacles, voir la scène de ce monde du music-hall, se reproduit sur le site pouvant même nous laisser dire que le spectacle a lieu sur Facebook. En ce sens, la translation qu'entraîne la mise en valeur n'implique pas forcément un changement de sens pour leurs acteurs. Mais les médiations sont aussi façonnées par les usages conventionnels de Facebook, aussi bien que par leurs contraintes techniques et morales. Les usagers remettent en scène un patrimoine avec lequel ils sont déjà familiarisés tout en actualisant leur regard ordinaire de fan; ils agissent ainsi sur la distance temporelle, spatiale et cognitive (Tornatore, 2006, 287) qui sépare leurs anciennes expériences in situ et leur évocation sur Facebook. Les groupes répondent à leurs désirs de mémoire en comblant une perte. Ils constituent donc un cadre pluriel de formes de subjectivation où chacun entretient un rapport émotionnel et pratique avec le passé et le présent, ainsi qu'avec les autres membres et El Paralelo. 
Bibliographie

AugéM. (1992). Non-lieux. Introduction à une anthropologie de la surmodernité, Paris, Seuil, $149 \mathrm{p}$.

Bensa A. (2001). «Fièvres d'histoire dans la France contemporaine». In Bensa A. et FabreD. (dir.), Une histoire à soi, Paris, Éditions de la Maison des Sciences de I'Homme, coll. Ethnologie de la France, cahier 18, p.1-13.

Caillé A. (2007). "Introduction ". In Caillé A. (dir.), La quête de la reconnaissance. Nouveau phénomène social total, Paris, La Découverte, coll. Textes à l'appui, p. 5-14.

Cardon D. (2008). «Présentation". In Réseaux, vol.6, n¹52, p.7-17.

Cardon D., Levrel J. (2009). "La vigilance participative. Une interprétation de la gouvernance de Wikipédia ". In Réseaux, vol.2, n¹54, p.51-89.

Cardon D., al (1995). "Parler en public». In Politix, vol. 8, n³1, p. 5-19.

Cheyronnaud J. (2006). «Fantastique d'un lieu têtu, l'Alcazar de Marseille". In Actes des journées AIBM, Groupe français de I'Association internationale des Bibliothèques, Archives et centres de documentation musicaux, no14, p.33-49.
Cheyronnaud J. (2011). "L'Alcazar de Marseille. Les termes d'une doxa ». In Cheyronnaud J., HureauS., Read V. (dir.), Les mondes du music-hall, Paris, Centre national du patrimoine de la chanson, des variétés et des musiques actuelles, http://www.lehall. com/galerie/musichall/?p=131

FabreD. (2013). «Le patrimoine porté par l'émotion ". In FabreD. (dir.), Émotions patrimoniales, Paris, Éditions de la Maison des Sciences de l'Homme, coll. Ethnologie de la France, cahier 27, p.13-98.

Fabiani J.-L. (2007). Après la culture légitime. Objets, publics, autorités, Paris, L'Harmattan, 257p.

Granovetter M. (1973). «The Strength of Weak Ties». In American Journal of Sociology, vol.78, n6 p.1360-1380.

Heinich N. (2009). La fabrique du patrimoine. "De la cathédrale à la petite cuillère», Paris, Éditions de la Maison des Sciences de l'Homme, coll. Ethnologie de la France, 286 p.

Honneth A. (2005).

"Invisibilité: Sur l'épistémologie de la 'reconnaissance'" . In Réseaux, vol.1, n¹29-130, p. 39-57

RicoeurP. (2000). La mémoire, I'histoire, I'oubli, Paris, Seuil, 689 p.

Tornatore J.-L. (2006). "Les formes d'engagement dans l'activité patrimoniale. De quelques manières de s'accommoder au passé". In V. Meyer \& J. Walter (dir.), Formes de l'engagement et espace public, 
Nancy, Presses

universitaires de Nancy, p.515-538.

Tornatore J.-L. (2006).

«Impressions patrimoniales.

Topologie de la perte et

photographie». In Jacques Roux et

Michel Peroni (dir.),

Sensibiliser. La sociologie dans le

vif du monde, La Tour d'Aigues,

Éditions de l'Aube, p.281-297. 
